\title{
Delay-minimal Transmission for Energy Constrained Wireless Communications
}

\author{
Jing Yang Sennur Ulukus \\ Department of Electrical and Computer Engineering \\ University of Maryland, College Park, MD 20742 \\ yangjing@umd.edu_ulukus@umd.edu
}

\begin{abstract}
We investigate the problem of minimizing the overall transmission delay of data packets in a single-user wireless communication system, where the transmitter has a fixed amount of energy to transmit all of the data packets. We consider two different scenarios. In the first scenario, we assume that packets arrive randomly at the transmitter. We propose two different approaches to solve this problem. First, we develop an iterative algorithm that allocates the total energy of the transmitter to its individual packets, in a way to minimize the total delay. As a second approach, we develop a dynamic programming formulation for the problem. In the second scenario, we assume that all of the packets have already arrived before the transmission starts. In this situation, the cost function has a fixed form, and is convex and differentiable. In this scenario, the iterative algorithm we develop is guaranteed to converge to the unique global optimal solution.
\end{abstract}

\section{INTRODUCTION}

For many wireless networks, especially for sensor and mobile ad-hoc networks, efficient consumption of energy is a critical issue, since energy is severely limited in such networks, and is even non-renewable in some cases. Consequently, a plethora of energy efficient methods and algorithms have been developed under various system settings and for various optimality criteria.

Reference [1] considers the problem of minimizing the energy used in transmitting packets in a single-user system subject to a deadline by which all packets must be transmitted. It develops an algorithm, coined lazy scheduling algorithm, where the transmission time of each packet is increased as much as possible, subject to the deadline and causality constraints (i.e., all packets must be transmitted by a deadline and a packet may not be transmitted before it arrives), in order to minimize the total energy consumed in transmitting the packets. In [2], the authors extend the single-user problem to a multi-user case and develop an iterative algorithm. The algorithm is named MoveRight where at any iteration only two consecutive packets are considered, and the starting points of the transmission of the two packets are moved to the right (hence the name). The authors prove that this algorithm converges to the optimal scheduling of the packets that minimizes the total energy. In [3], the authors extend their energyminimizing approaches to wider scenarios, such as multipleaccess and broadcast channels, and to channels with fading.

This work was supported by NSF Grants CCF 04-47613, CCF 05-14846, CNS 07-16311 and CCF 07-29127.
Dynamic programming (DP) is another approach which has been broadly adopted in wireless optimal power/energy allocation problems. In [4], the authors use DP to jointly optimize the long-term average transmission power and average buffer delay. Reference [5] proposes a DP formulation to find optimal power, channel coding and source coding policies with a delay constraint. Reference [6] uses DP to maximize the $K$ block constrained capacity through adaptively allocating power across blocks according to the varying channel condition. Reference [7] uses DP to calculate the optimal power/rate control policies to minimize the average delay under an average power constraint. Reference [8] uses DP to solve the problem of maximizing the data throughput for an energy- and deadline-constrained transmitter over a fading channel.

In this paper, we consider the "dual" of the problem considered in [1]. For a single-user system, our objective is to minimize the overall delay of the packets subject to an energy constraint on the transmitter. The delay includes both the queuing time and the transmission time for the packet. Our aim is to allocate the total energy over all packets in a way to minimize the overall delay of the system. We investigate two different settings. In the first setting, we assume that the packets arrive randomly during the transmissions, while in the second setting, we assume that all of the packets have arrived and are ready for transmission before the transmission starts. We formulate both problems as convex minimization problems. However, we see that there are important structural differences between the two problems.

In the first setting, even though the overall cost function is convex in the energies allocated to the packets, it is not differentiable. The reason for this is that the cost function takes different forms in different regions of allowable energy distributions. In other words, the energy allocated to a packet affects the form of the cost function for later packets. In the second setting, the cost function is convex, has a fixed form, and is differentiable. For the first setting, unlike [1], our problem does not admit a closed-form solution. Therefore, we develop an iterative algorithm that is based on the principle of decreasing the overall delay at each iteration. We prove that the proposed algorithm decreases the overall delay monotonically. However, due to the non-differentiability of the overall delay function, the proposed algorithm may converge to a suboptimal fixed point. In order to overcome this problem, we use two modifications on our algorithm: increasing the dimensionality of the sub-problem solved at each iteration (i.e., considering more than two packets at any given iteration), 


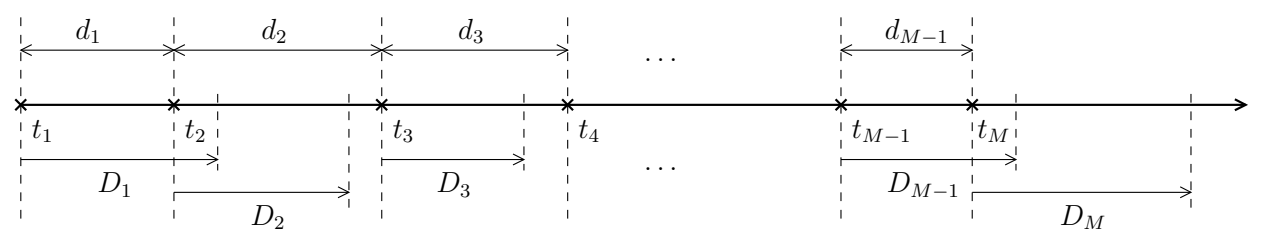

Fig. 1. System model with random packet arrivals.

and $\epsilon$-perturbation of the sub-optimal fixed points. In addition, we develop a DP based formulation for the same problem. For the second setting, we formulate the standard Lagrangian based approach, and also develop an iterative algorithm. Since the cost function has a fixed form and is differentiable, the iterative algorithm is guaranteed to converge to the unique global optimum solution.

\section{SCENARIO I: RANDOM PACKET ARRIVALS}

We consider a non-fading single-user wireless channel. We assume that $M$ packets arrive at the transmitter randomly at times $t_{1}, t_{2}, \ldots, t_{M}$, where the inter-arrival times are denoted as $d_{1}, d_{2}, \ldots, d_{M-1}$; see Fig. 1 . The transmitter has a total energy constraint which is denoted by $E$. Let $e_{i}$ denote the energy allocated for the transmission of packet $i$, then $\sum_{i=1}^{M} e_{i} \leq E$. Since the channel is non-fading, we can express the relationship between the transmission duration of $\tau_{i}$ and the energy spent in its transmission $e_{i}$, for packet $i$, as a deterministic function $\tau_{i}=f\left(e_{i}\right)$. Without loss of generality, as in [1], [3], we assume that $f(e)$ satisfies the following properties: i) $f(e) \geq 0$, ii) $f(e)$ decreases monotonically in $e$, iii) $f(e)$ is strictly convex in $e$, iv) $f(e)$ is continuously differentiable, and v) $f(e) \rightarrow \infty$ as $e \rightarrow 0$. As shown in [1], [3], the first four conditions are satisfied in realistic channel coding schemes. The last condition is reasonable as a packet cannot be transmitted with zero energy.

Let $D_{i}$ denote the delay experienced by the $i$-th packet, which includes the waiting time in the queue and the transmission time. Then, the delay experienced by each packet can be written recursively as,

$$
\begin{aligned}
D_{1} & =f\left(e_{1}\right) \\
D_{2} & =\left(D_{1}-d_{1}\right)^{+}+f\left(e_{2}\right) \\
D_{3} & =\left(D_{2}-d_{2}\right)^{+}+f\left(e_{3}\right) \\
& \vdots \\
D_{M} & =\left(D_{M-1}-d_{M-1}\right)^{+}+f\left(e_{M}\right)
\end{aligned}
$$

where $(x)^{+}=\max (0, x)$. Here, for the $i^{\text {th }}$ packet, $\left(D_{i-1}-\right.$ $\left.d_{i-1}\right)^{+}$denotes the waiting time in the queue, and $f\left(e_{i}\right)$ denotes the actual transmission time. Then, we can express our optimization problem as

$$
\begin{array}{ll}
\min & \sum_{i=1}^{M} D_{i} \\
\text { s.t. } & \sum_{i=1}^{M} e_{i} \leq E \\
& e_{i} \geq 0, \quad i=1,2, \ldots, M
\end{array}
$$

where the parameters of the optimization are the energies allocated to all packets, $\left\{e_{i}\right\}_{i=1}^{M}$, and the givens of the optimization problem are the total energy $E$ and the inter-arrival times of the packets $\left\{d_{i}\right\}_{i=1}^{M}$.

Intuitively, the optimization problem in (2) is a convex optimization problem since function $f\left(e_{i}\right)$ is convex and a linear combination of convex functions is convex. However, the existence of $(\cdot)^{+}$function complicates matters, and the joint convexity of the cost function with respect to all $e_{i}$, i.e., with respect to $\mathbf{e}=\left[\begin{array}{llll}e_{1} & e_{2} & \ldots & e_{M}\end{array}\right]^{\top}$ needs to be proved.

Theorem 1: The objective function in (2) is convex with respect to $\mathbf{e}$.

Proof: We will prove the convexity recursively. First, we note that $D_{1}=f\left(e_{1}\right)$ and $f\left(e_{1}\right)$ is convex in $e_{1}$. We also note that $D_{2}=\left(f\left(e_{1}\right)-d_{1}\right)^{+}+f\left(e_{2}\right)$ and the function $\left(f\left(e_{1}\right)-d_{1}\right)^{+}$is convex in e because of the convexity of the function $f\left(e_{1}\right)$ in $e_{1}$. Thus, $D_{2}$ is convex in e also.

Then, we look at $D_{3}=\left(\left(f\left(e_{1}\right)-d_{1}\right)^{+}+f\left(e_{2}\right)-d_{2}\right)^{+}+$ $f\left(e_{3}\right)$. We let $F(\mathbf{e})=\left(f\left(e_{1}\right)-d_{1}\right)^{+}+f\left(e_{2}\right)-d_{2}$. We note that $F(\mathbf{e})$ itself is convex in e, and we need to prove that $(F(\mathbf{e}))^{+}$is convex in $\mathbf{e}$ as well. Using the definition of $(\cdot)^{+}$, for any two vectors $\mathbf{e}$ and $\mathbf{e}^{\prime}$ in the constraint set, we have

$$
\begin{aligned}
\lambda F(\mathbf{e})^{+}+(1-\lambda) F\left(\mathbf{e}^{\prime}\right)^{+} & \geq \lambda F(\mathbf{e})+(1-\lambda) F\left(\mathbf{e}^{\prime}\right) \\
& \geq F\left(\lambda \mathbf{e}+(1-\lambda) \mathbf{e}^{\prime}\right)
\end{aligned}
$$

If $F\left(\lambda \mathbf{e}+(1-\lambda) \mathbf{e}^{\prime}\right)$ is positive, we have

$$
\begin{aligned}
\lambda F(\mathbf{e})^{+}+(1-\lambda) F\left(\mathbf{e}^{\prime}\right)^{+} & \geq F\left(\lambda \mathbf{e}+(1-\lambda) \mathbf{e}^{\prime}\right) \\
& =F\left(\lambda \mathbf{e}+(1-\lambda) \mathbf{e}^{\prime}\right)^{+}
\end{aligned}
$$

If $F\left(\lambda \mathbf{e}+(1-\lambda) \mathbf{e}^{\prime}\right)$ is negative, then $F\left(\lambda \mathbf{e}+(1-\lambda) \mathbf{e}^{\prime}\right)^{+}=0$. Using the nonnegativity of the $(\cdot)^{+}$function, we have

$$
\begin{aligned}
\lambda F(\mathbf{e})^{+}+(1-\lambda) F\left(\mathbf{e}^{\prime}\right)^{+} & \geq 0 \\
& =F\left(\lambda \mathbf{e}+(1-\lambda) \mathbf{e}^{\prime}\right)^{+}
\end{aligned}
$$

Therefore, using (4) and (5), we conclude that

$$
\lambda F(\mathbf{e})^{+}+(1-\lambda) F\left(\mathbf{e}^{\prime}\right)^{+} \geq F\left(\lambda \mathbf{e}+(1-\lambda) \mathbf{e}^{\prime}\right)^{+}
$$

which implies that $(F(\mathbf{e}))^{+}$is convex in e. Therefore, $D_{3}$ is convex in e as well.

The convexity of $\left(D_{i}-d_{i}\right)^{+}$for $i=4, \ldots, M-1$ can be proved in a similar manner. Since the objective function can be expressed as

$$
\sum_{i=1}^{M} D_{i}=\sum_{i=1}^{M-1}\left(D_{i}-d_{i}\right)^{+}+\sum_{i=1}^{M} f\left(e_{i}\right)
$$

and since each term in the cost function is convex in $\mathbf{e}$, the linear combination is convex in e as well.

Therefore, our problem is a convex minimization problem which has a convex objective function and linear constraints. However, there are two main difficulties in this optimization 
problem. First, since the overall delay includes both the queuing time and the transmission time of the packets, the transmission time for a packet affects the queuing time of all of the following packets. This causes the queuing time of earlier packets to be multiply counted in the objective function. This leads to the varying coefficients before $f\left(e_{i}\right)$ 's in the cost function, which implies that the convexity of $f(\cdot)$ alone will not provide us a closed-form solution; we note that the convexity of the cost function alone provided a closed-form solution in [1] due to the symmetry in the cost function. Secondly, because of the existence of $(\cdot)^{+}$function in the overall delay expression, the cost function has nondifferentiable points. In addition, depending on whether the insides of $(\cdot)^{+}$functions are negative or positive, we have $2^{M}$ possible cost functions. Since the number of different cost functions to consider grows exponentially with the number of packets, standard Lagrangian method is not tractable here. In the following, we will use a simple 3-packet problem to illustrate the difficulties involved in solving this convex optimization problem.

Using the definition of $D_{i}$ in (1), the 3-packet problem is

$$
\begin{array}{ll}
\min & f\left(e_{1}\right)+\left(f\left(e_{1}\right)-d_{1}\right)^{+}+f\left(e_{2}\right)+ \\
& \left(\left(f\left(e_{1}\right)-d_{1}\right)^{+}+f\left(e_{2}\right)-d_{2}\right)^{+}+f\left(e_{3}\right) \\
\text { s.t. } & e_{1}+e_{2}+e_{3} \leq E, \quad e_{1}, e_{2}, e_{3} \geq 0
\end{array}
$$

Opening the parentheses, we have four different possible cases:

Case 1: Both the transmission of the first and second packets end before the arrival of the next packet, i.e., insides of both $(\cdot)^{+}$functions are negative. This case is illustrated in Fig. 2. In this case, we have

$$
\begin{array}{cl}
\min & f\left(e_{1}\right)+f\left(e_{2}\right)+f\left(e_{3}\right) \\
\text { s.t. } & f\left(e_{1}\right) \leq d_{1}, \quad f\left(e_{2}\right) \leq d_{2} \\
& e_{1}+e_{2}+e_{3} \leq E, \quad e_{1}, e_{2}, e_{3} \geq 0
\end{array}
$$

Case 2: The transmission of the first packet ends after the arrival of the second packet, while the transmission of the second packet ends before the arrival of the third packet. This case is illustrated in Fig. 3. In this case, we have

$$
\begin{array}{cl}
\min & 2 f\left(e_{1}\right)+f\left(e_{2}\right)+f\left(e_{3}\right)-d_{1} \\
\mathrm{s.t.} & f\left(e_{1}\right)>d_{1}, \quad f\left(e_{1}\right)+f\left(e_{2}\right) \leq d_{1}+d_{2} \\
& e_{1}+e_{2}+e_{3} \leq E, \quad e_{1}, e_{2}, e_{3} \geq 0
\end{array}
$$

Case 3: The transmission of the first packet ends before the arrival of the second packet, while the transmission of the second packet ends after the arrival of the third packet. This case is illustrated in Fig. 4. In this case, we have

$$
\begin{array}{cl}
\min & f\left(e_{1}\right)+2 f\left(e_{2}\right)+f\left(e_{3}\right)-d_{2} \\
\text { s.t. } & f\left(e_{1}\right) \leq d_{1}, \quad f\left(e_{2}\right)>d_{2} \\
& e_{1}+e_{2}+e_{3} \leq E, \quad e_{1}, e_{2}, e_{3} \geq 0
\end{array}
$$

Case 4: The transmissions of both the first and the second packets end after the arrival of the next packet. This case is

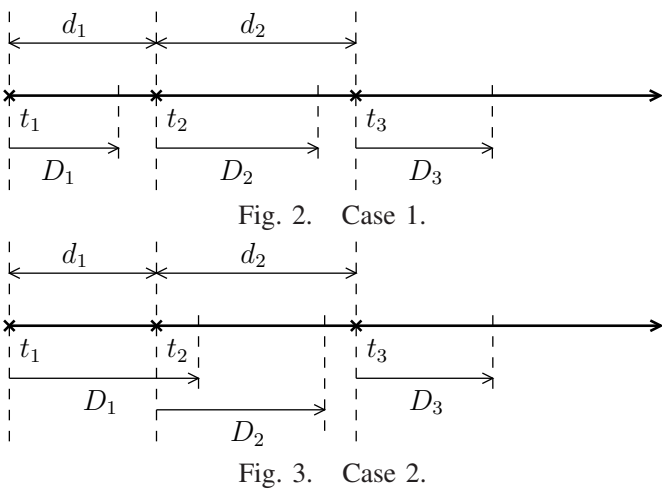

illustrated in Fig. 5. In this case, we have

$$
\begin{array}{cl}
\min & 3 f\left(e_{1}\right)+2 f\left(e_{2}\right)+f\left(e_{3}\right)-2 d_{1}-d_{2} \\
\mathrm{s.t.} & f\left(e_{1}\right)>d_{1}, \quad f\left(e_{1}\right)+f\left(e_{2}\right)>d_{1}+d_{2} \\
& e_{1}+e_{2}+e_{3} \leq E, \quad e_{1}, e_{2}, e_{3} \geq 0
\end{array}
$$

As we see, the sub-problems in (9), (10), (11) and (12) are similar in structure, except for different coefficients in front of the transmission delay times, $f\left(e_{i}\right)$, in the cost function. In addition, each problem has a different constraint set, which are all convex due to the monotonicity of $f\left(e_{i}\right)$ in $e_{i}$. In order to solve the optimization problem in (8), we need to solve the four optimization problems in (9), (10), (11) and (12), and take the solution that gives us the smallest cost function, i.e., overall delay. Even though each problem is differentiable and convex, the number of problems to be solved increases exponentially with the number of packets, making this approach intractable for practical scenarios with many packet arrivals.

\section{A. An Iterative Approach}

Because of the intractability of the global problem, in this section, we consider developing an iterative algorithm, which at any given iteration, considers a smaller local sub-problem. Similar to the FlowRight algorithm developed in [3], in this section, we consider optimizing two of the variables, the energies allocated to two consecutive packets, at any iteration, when the rest of the variables, the energies allocated to the rest of the packets, are fixed.

Initially, we allocate the total energy $E$ to the first packet. Then, we consider the first two packets, and optimize the distribution of the total energy $E$ over these two packets, in a way to minimize the overall delay, while we keep the energies allocated to the rest of the packets fixed. We continue this process until we reach the last packet, then we return to the first packet. We express the local optimization problem in terms of the energies of two consecutive packets, as follows

$$
\begin{array}{ll}
\min & \sum_{j=i}^{M} D_{j}\left(e_{i}^{k}, e_{i+1}^{k}\right) \\
\text { s.t. } & e_{i}^{k}+e_{i+1}^{k}=e_{i}^{k-1}+e_{i+1}^{k-1}, \quad e_{i}^{k}, e_{i+1}^{k} \geq 0
\end{array}
$$

where $e_{i}^{k-1}$ and $e_{i+1}^{k-1}$ denote the energies of the packets in the previous iteration. This problem can be solved relatively easily as it essentially is a single-variable optimization problem.

It is easy to prove that this algorithm converges to a fixed point, since the algorithm monotonically decreases the cost 


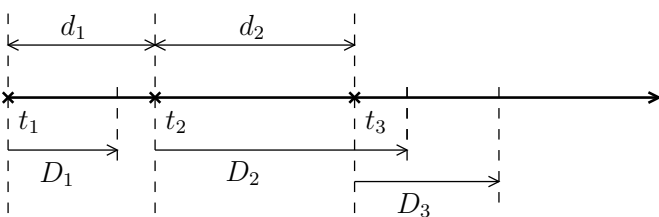

Fig. 4. Case 3.

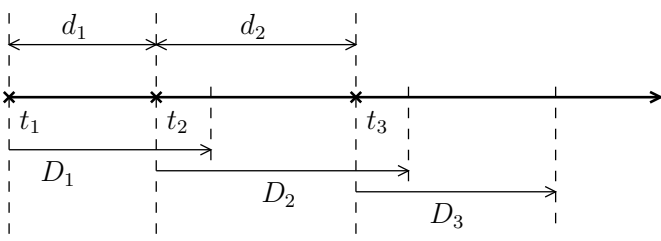

Fig. 5. Case 4 .

function which is lower bounded by zero. If the objective function was twice-differentiable, we could be sure that the algorithm converges to the globally optimum solution. Since our cost function is not differentiable at some points, the algorithm may converge to a strictly sub-optimal fixed point. Reference [9] proposes two approaches to solve the difficulty introduced by non-differentiability in network flow problems: "multiple node relaxation method", and " $\epsilon$-relaxation method". We adopt these two methods here in order to escape sub-optimal fixed points. Following multiple node relaxation method, we consider sub-problems involving three or more packets, as opposed to two packets as we have done above. Similarly, following the $\epsilon$-relaxation method, we move a small amount of energy from one packet to another to perturb a suboptimal fixed point. Experimentally, we have observed that both methods improve the convergence of the algorithm.

\section{B. A Dynamic Programming Approach}

In this section, we develop a DP approach to our delay minimization problem. In particular, we partition the problem into $M$ stages, and define the state space to be $\mathcal{E} \times \mathcal{A}$, where $\mathcal{E}$ includes the possible amounts of energy remaining at the current stage and $\mathcal{A}$ is the set of possible queuing times associated with the packet. Specifically, in stage $n$, we define $S_{n}(e, a)$ to be the minimal delay for the last $M-n$ packets, given the total energy remaining is $e$ and the waiting time in the queue for the $n$-th packet is $a$, as shown in Fig. 6 . Then, we have the following recursive relationship

$$
\begin{aligned}
S_{n}(e, a)=\min _{0 \leq e_{n} \leq e}\left\{a+f\left(e_{n}\right)\right. & \\
+ & \left.S_{n+1}\left(e-e_{n},\left(a+f\left(e_{n}\right)-d_{n}\right)^{+}\right)\right\}
\end{aligned}
$$

for $n=1,2, \ldots, M-1$, and $S_{M+1}(e, a)=0$.

During the process of solving the recursive equations backwards, we keep track of $e_{n}$ that leads to the minimum value. Let us denote the minimizing values as $\hat{e}_{n}(e, a)$ for $n=1,2, \ldots, N$.

After computing the functions $\left\{S_{n}(e, a), 0 \leq e \leq E\right\}$ in a backward recursion and obtaining the $\hat{e}_{n}(e, a)$, we get the optimal energy allocation strategy as $e_{1}=\hat{e}_{1}(E, 0)$. For $n=$ $2, \ldots, N$,

$$
\begin{aligned}
& a_{n}=\left(a_{n-1}+f\left(e_{n-1}\right)-d_{n-1}\right)^{+} \\
& e_{n}=\hat{e}_{n}\left(E-\sum_{i=1}^{n-1} e_{i}, a_{i}\right)
\end{aligned}
$$

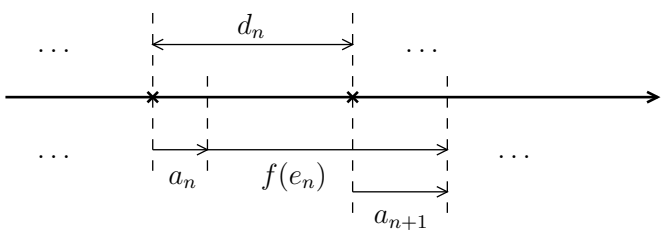

Fig. 6. System model for the dynamic programming approach.

Since getting a closed form solution for the recursive equations appears to be intractable, we perform numerical approximation instead. To this end, we quantize the state space into a finite number of discrete states. The step size of the quantization decides the size of the state space. Specifically, if there are $N$ levels for the energy and $J$ levels for the waiting time, for each packet we have $N \cdot J$ different states. The number of evaluations of $a+f\left(e_{n}\right)+S_{n+1}\left[e-e_{n},(a+\right.$ $\left.\left.f\left(e_{n}\right)-d_{n}\right)^{+}\right]$is once per quantized $e_{n}$ for each quantized state for each stage. Thus, the number of basic evaluations is $N^{2} J M$, and the number of calculations grows linearly with the total number of packets $M$. We note that we can use the DP approach for more general cases where the packet arrivals are modeled as a random process, and the delays are calculated as expectations. In addition, we can incorporate the fading nature of the wireless channel, as well as develop online algorithms.

\section{SCEnARio II: PACKets ReAdy Before TRANSMISSION STARTS}

In many situations, such as multimedia communications, the source (video, music, etc.) may be available at the server waiting to be downloaded to their destinations. In sensor networks, a node may have gathered a number of packets before the transmission starts. In these scenarios, minimizing the overall transmission delay with a given amount of energy is also an important problem.

We assume that there are $M$ packets available at the transmitter at $t=0$; see Fig. 7. Therefore, for the $i$-th packet, the delay $D_{i}$ can be expressed as

$$
D_{i}=\sum_{k=1}^{i} \tau_{k}=\sum_{k=1}^{i} f\left(e_{k}\right)
$$

Then, our optimization problem becomes

$$
\begin{array}{ll}
\min & \sum_{i=1}^{M}(M-i+1) f\left(e_{i}\right) \\
\text { s.t. } & \sum_{i=1}^{M} e_{i} \leq E \\
& e_{i} \geq 0, \quad i=1, \ldots, M
\end{array}
$$

We note that, since all the packets have arrived before the transmission starts, the cost function has a fixed form, and we do not need to use the $(\cdot)^{+}$function. This makes the optimization problem tractable. The problem in (17) is a convex optimization problem, and there exists a unique global optimum solution that satisfies the KKT optimality conditions.

We note that because of property v) of $f(e)$, no $e_{i}$ can be zero, as it would require the cost function to go to infinity. As a result, the KKTs can be expressed as

$$
(M-i+1) f^{\prime}\left(e_{i}\right)+\lambda=0
$$




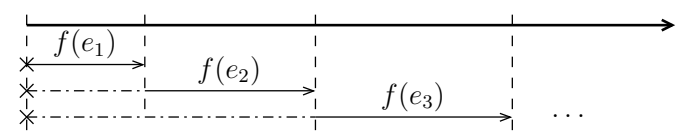

Fig. 7. System model when all packets are ready before the transmission starts.

i.e., as

$$
e_{i}=f^{\prime-1}\left(\frac{-\lambda}{M-i+1}\right), \quad i=1,2, \ldots, M
$$

where $\lambda$ is the non-negative Lagrange multiplier which is chosen such that $\sum_{k=1}^{i} e_{k}=E$.

In the following, we also devise an iterative algorithm to solve this problem. Following the procedure described in the previous section, considering two variables at a time, the local optimization in the $k$-th iteration becomes

$$
\begin{array}{cl}
\min & (M-i+1) f\left(e_{i}^{k}\right)+(M-i) f\left(e_{i+1}^{k}\right) \\
\text { s.t. } & e_{i}^{k}+e_{i+1}^{k}=e_{i}^{k-1}+e_{i+1}^{k-1}, \quad e_{i}^{k}, e_{i+1}^{k} \geq 0
\end{array}
$$

Following a similar analysis as before, we know that $\mathbf{e}^{k}$ converges to a fixed point, $\overline{\mathbf{e}}$, as the cost function is monotonically decreasing and is lower bounded. We need to show that $\overline{\mathbf{e}}$ is the solution to (17). From the KKTs of the local optimization, we have

$$
M f^{\prime}\left(\bar{e}_{1}\right)=(M-1) f^{\prime}\left(\bar{e}_{2}\right)=\ldots=f^{\prime}\left(\bar{e}_{M}\right)
$$

We also have $\sum_{i=1}^{M} \bar{e}_{i}=E$. Therefore, $\overline{\mathbf{e}}$ satisfies the global KKT conditions in (18) and is the globally optimal point.

Based on the properties of $f(e)$, we know that $f^{\prime}(e)$ is negative and monotonically increasing in $e$. From (21), we have $f^{\prime}\left(\bar{e}_{1}\right)>f^{\prime}\left(\bar{e}_{2}\right)>\ldots>f^{\prime}\left(\bar{e}_{M}\right)$. Therefore, at the optimal point, the energy spent for each packet monotonically decreases in the order of transmission. Thus, earlier packets are assigned larger energies and therefore, are transmitted quicker than the later ones. Therefore, this model for the delay minimization problem yields a solution which is in contrast with the principle of lazy scheduling that the model in [1] resulted in.

\section{Simulation Results}

We generate a short sequence of packets, with inter-arrival times $\mathbf{d}=\left[\begin{array}{llll}1 & 0.5 & 1.5 & 1.75\end{array}\right]$. The first packet arrives at time $t=0$. We choose a simple function to use in our simulations: $f(e)=1 / e$, which satisfies all of the stated properties for $f(e)$. We use five algorithms, including our iterative algorithm, the versions of it with dimension relaxation, and $\epsilon$ perturbation methods, DP based algorithm and built-in Matlab optimization functions.

Simulation results indicate that DP based algorithm always converges to the solution that the built-in Matlab function finds. In Fig. 8, we observe that our iterative algorithm converges to the solution the built-in Matlab function finds. However, in Fig. 9, we observe that there is a gap between the convergence point of our iterative algorithm and the Matlab solution. We note that, at the point that our algorithm converges to, the departure time of the third packet coincides with the arrival time of the fourth packet. This means that our algorithm converges to a non-differentiable sub-optimal

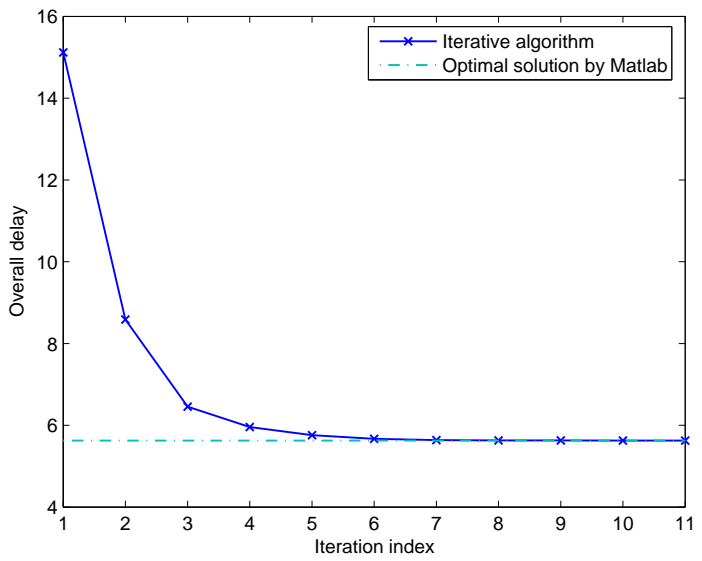

Fig. 8. Overall delay as a function of the iteration index, when $E=4.8$.

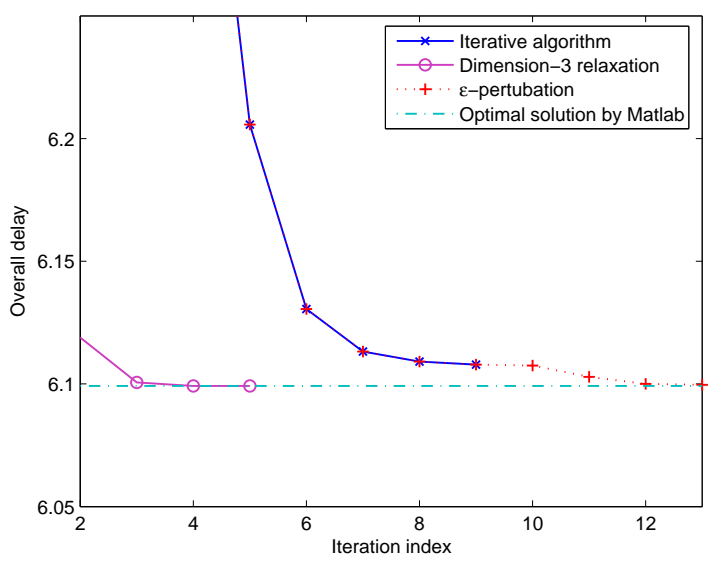

Fig. 9. Overall delay as a function of the iteration index, when $E=4.5$

fixed point. When we apply dimension-3 relaxation and $\epsilon$ perturbation methods, we observe that the modified version of our algorithm escapes the sub-optimal fixed point and converges to the optimal solution.

\section{REFERENCES}

[1] E. Uysal-Biyikoglu, B. Prabhakar, and A. El Gamal, "Energy-efficient packet transmission over a wireless link," IEEE/ACM Transactions on Networking, vol. 10, pp. 487-499, 2002.

[2] A. El Gamal, C. Nair, B. Prabhakar, E. Uysal-Biyikoglu, and S. Zahedi, "Energy-efficient scheduling of packet transmissions over wireless networks," IEEE Infocom, vol. 3, pp. 1773-1782, 2002.

[3] E. Uysal-Biyikoglu and A. El Gamal, "On adaptive transmission for energy efficiency in wireless data networks," IEEE Transactions on Information Theory, vol. 50, pp. 3081-3094, Dec 2004.

[4] R. A. Berry and R. G. Gallager, "Communication over fading channels with delay constraints," IEEE Transactions on Information Theory, vol. 48, pp. 1135-1149, May 2006.

[5] T. Holliday and A. Goldsmith, "Optimal power control and sourcechannel coding for delay constrained traffic over wireless channels," Proc. IEEE Int. Conf. Communications, vol. 2, pp. 831-835, Apr/May 2002.

[6] R. Negi and J. M. Cioffi, "Delay-constrained capacity with casual feedback," IEEE Transactions on Information Theory, vol. 48, pp. 24782494, Sep 2003.

[7] I. Bettesh and S. Shamai, "Optimal power and rate control for minimal average delay: The single-user case," IEEE Transactions on Information Theory, vol. 52, pp. 4115-4141, Sep 2006.

[8] A. Fu, E. Modiano, and J. Tsitsiklis, "Optimal energy allocation for delay-constrained data transmission over a time-varying channel," IEEE Infocom, vol. 2, pp. 1095-1105, 2003.

[9] D. Bertsekas and J. Tsitsiklis, Parallel and Distributed Computation: Numericcal Methods. Athena Scientific, 1997. 